\title{
Predicting postoperative hypertension among patients with ventricular septal defect in infants.
}

\author{
Junpei Kawamura ${ }^{1}$, Kentaro Ueno ${ }^{1}$, Tsubasa Shimozono ${ }^{1}$, Yoshihiro Takahashi ${ }^{1}$, Koji \\ Nakae $^{1}$, and Yasuhiro Okamoto ${ }^{1}$ \\ ${ }^{1}$ Affiliation not available
}

September 25, 2021

\begin{abstract}
Background: Patients with strong pulmonary vascular occlusive lesions are at risk of developing postoperative pulmonary hypertension $(\mathrm{PH})$. We aimed to evaluate preoperative right ventricular (RV) function in patients with ventricular septal defect (VSD) who required cardiac surgery during infancy and consequently developed postoperative PH and to determine whether we could preliminarily evaluate postoperative $\mathrm{PH}$ in these patients. Methods: We retrospectively analyzed 55 infants with VSD who underwent cardiac surgery between March 2014 and April 2020. We evaluated the measurements of preoperative general function and 2D strain between these two groups: a group with postoperative $\mathrm{PH}$ (post- $\mathrm{PH}, \mathrm{n}=10$ ) and a group without postoperative PH (post-NPH, n=45). Results: Post-PH patients had a significantly lower tricuspid annular plane systolic excursion (TAPSE) $(11.1 \mathrm{~mm})$, TAPSERA (the proportion of TAPSE due to right atrial (RA) contraction alone) (7.0 mm), RA ejection fraction $(36.1 \%)$ and RA expansion index $(56.4 \%)$ than the post-NPH patients. Furthermore, the post-PH group had a significantly lower peak RA longitudinal strain (PRALS) $(32.0 \%)$ than the post-NPH group (43.0\%). Multivariate logistic regression model demonstrated that PRALS was independent echocardiographic parameters for the presence of post-PH (OR 1.18, $95 \%$ CI: $1.02-1.36, \mathrm{p}=0.03)$. The sensitivity and specificity of predicting post-PH for [?] $35 \%$ of the PRALS were $88.9 \%$ and $70.0 \%$, respectively, with an area under the curve of $0.85(\mathrm{p}<0.01)$. Conclusion: RA parameters demonstrated preoperative RV diastolic dysfunction in the post-PH group. PRALS could be useful factors for predicting postoperative PH.
\end{abstract}

\section{Original Investigation}

Predicting postoperative hypertension among patients with ventricular septal defect in infants.

Junpei Kawamura ${ }^{1}$, MD, Kentaro Ueno ${ }^{1^{*}}$, phD, Tsubasa Shimozono ${ }^{1}$, MD, Yoshihiro Takahashi ${ }^{1}$, MD, Koji Nakae $^{1}$, MD, Yasuhiro Okamoto ${ }^{1}$, phD

Department of Pediatrics, Kagoshima University Graduate School of Medical and Dental Sciences, Kagoshima, Japan

Running title: Predicting postoperative hypertension in infants.

*Corresponding author: Kentaro Ueno

Kagoshima University Graduate School of Medical and Dental Sciences

8-35-1 Sakuragaoka, Kagoshima 890-8544, Japan

Tel: 099-275-5354, Fax: 099-265-7196

Email: ukenta@m3.kufm.kagoshima-u.ac.jp

Abstract 
Background: Patients with strong pulmonary vascular occlusive lesions are at risk of developing postoperative pulmonary hypertension $(\mathrm{PH})$. We aimed to evaluate preoperative right ventricular (RV) function in patients with ventricular septal defect (VSD) who required cardiac surgery during infancy and consequently developed postoperative $\mathrm{PH}$ and to determine whether we could preliminarily evaluate postoperative $\mathrm{PH}$ in these patients.

Methods: We retrospectively analyzed 55 infants with VSD who underwent cardiac surgery between March 2014 and April 2020. We evaluated the measurements of preoperative general function and 2D strain between these two groups: a group with postoperative $\mathrm{PH}$ (post- $\mathrm{PH}, \mathrm{n}=10$ ) and a group without postoperative $\mathrm{PH}$ (post-NPH, $\mathrm{n}=45$ ).

Results: Post-PH patients had a significantly lower tricuspid annular plane systolic excursion (TAPSE)

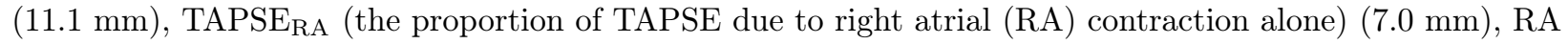
ejection fraction $(36.1 \%)$ and RA expansion index $(56.4 \%)$ than the post-NPH patients. Furthermore, the post-PH group had a significantly lower peak RA longitudinal strain (PRALS) $(32.0 \%)$ than the postNPH group (43.0\%). Multivariate logistic regression model demonstrated that PRALS was independent echocardiographic parameters for the presence of post-PH (OR 1.18, 95\% CI: $1.02-1.36, p=0.03$ ) . The sensitivity and specificity of predicting post-PH for [?] $35 \%$ of the PRALS were $88.9 \%$ and $70.0 \%$, respectively, with an area under the curve of $0.85(\mathrm{p}<0.01)$.

Conclusion: RA parameters demonstrated preoperative RV diastolic dysfunction in the post-PH group. PRALS could be useful factors for predicting postoperative PH.

Keywords: ventricular septal defect, echocardiography, pulmonary hypertension, pediatrics, right atrial strain

\section{Introduction}

In patients with a congenital heart disease with an uncorrected left-to-right shunt, such as ventricular septal defect (VSD), increased pulmonary pressure leads to vascular remodeling and dysfunction, resulting in a progressive increase in pulmonary vascular resistance and an increased pressure in the right chambers of the heart [1]. Therefore, patients with strong pulmonary vascular occlusive lesions are at risk for developing postoperative pulmonary hypertension (PH) [2]. Certain conditions such as Down syndrome (DS) are often associated with pulmonary vascular obstructive lesions and subsequently can cause postoperative $\mathrm{PH}$ [3]. Although home oxygen therapy and pulmonary vasodilators are effective treatments for prolonged postoperative PH after surgery for congenital heart defects [4, 5], PH still significantly impairs patients' exercise capacity and quality of life. Postoperative $\mathrm{PH}$ is often diagnosed based on the peak velocity of the pulse-Doppler in tricuspid regurgitation on echocardiography and pulmonary artery pressure on cardiac catheterization. However, it is difficult to predict postoperative PH after cardiac surgery in patients with VSD because their preoperative characteristics vary. In infants with large VSDs, preoperative RV stress findings are difficult to discern from symptoms due to shunting. In cases of severe idiopathic PH, RV function is impaired due to the high vascular resistance caused by pulmonary vascular occlusive lesions [6, 7]. Similarly, in infants and children with PH, such as that seen in DS, RV contractility and diastolic capacity are impaired $[8,9]$. We hypothesized that the right ventricle may be under pressure and the RV function may be impaired preoperatively in patients with a large VSD with strong pulmonary vascular occlusive lesions. In our study, we used echocardiography to evaluate the preoperative RV function in patients with VSD who required surgery during early infancy and determined whether we could preliminarily predict their development of postoperative $\mathrm{PH}$.

\section{Methods}

The Kagoshima University Ethics Committee approved this study, which was performed in accordance with the International Conference on Harmonization Good Clinical Practice Guidelines, and the Declaration of Helsinki. All procedures involving human participants followed the ethical standards of the Institutional and/or National Research Committee (Kagoshima University Ethics Committee, 190055). The requirement 
for parental/patient informed consent was waived.

Subjects

We investigated retrospectively 61 patients with VSD underwented surgery between March 2014 and April 2020, within their first year of life at the Department of Cardiovascular Surgery, Kagoshima University Hospital. Among these patients, five were excluded in accordance with the study's exclusion criteria, leaving 55 patients included in the study. The exclusion criteria were as follows: patients with developmental lung disorder, air tract narrowing, other cardiac diseases such as atrial septal defect (ASD), patent ductus arteriosus, pulmonary artery stenosis, chromosomal abnormalities other than DS, and having a postoperative significant VSD leakage. Preoperative indications for surgery were as follows: 1) having a left ventricular (LV) volume overload or [?] $1.5 \%$ of the estimated pulmonary to systemic blood flow ratio or 2) having a the non-restrictive VSD estimating preoperative high pressure of RV on echocardiography. We retrospectively analyzed the results based on medical and laboratory records.

We examined the results by dividing the patients into two groups based on the findings of the postoperative echocardiography: the group with postoperative $\mathrm{PH}$ (post-PH) and the group without postoperative $\mathrm{PH}$ (post-NPH). Postoperative measurements were taken 1-3 months after the VSD surgery in all patients. Post-PH was defined as a patient with a tricuspid regurgitation pressure gradient (TRPG) [?] $27 \mathrm{mmHg}$ after surgery, with reference to three-quarters of $36 \mathrm{mmHg}$, which is the cutoff for TRPG in adult PH studies [10].

Preoperative 2D echocardiographic measurements

Preoperative measurements were taken in all patients within 3 months of VSD surgery. The VSD diameter $(\mathrm{mm})$ of the aortic valve in the short-axis view and the trans-VSD pressure gradient (VSD PG, $\mathrm{mmHg}$ ) were calculated using the simplified Bernoulli equation with the pulse-Doppler of the blood flow through the VSD. Echocardiographic measurements of the left atrial and ventricular system included the following: left ventricular diastolic diameter per body surface area (LVDd/BSA, $\mathrm{mm} / \mathrm{m}^{2}$ ), left ventricular fractional shortening (LVFS, \%), left atrial volume index (LAVI, $\mathrm{cm}^{2} / \mathrm{m}^{2}$ ), left atrial total ejection fraction (LA EF, $\%$ ), and left atrial expansion index (LA expansion index, \%) in the four-chamber view.

Echocardiographic measurements to assess the right atrial and ventricular system included the following: RV fractional area change (RV FAC, \%), tricuspid annular plane systolic excursion (TAPSE, mm), right atrial area volume index (RAVI, $\mathrm{cm}^{2} / \mathrm{m}^{2}$ ), right atrial ejection fraction (RAEF, \%), and right atrial expansion index (RA expansion index,\%) in the four-chamber view. TAPSE $\mathrm{RA}_{\mathrm{A}}$ (proportion of TAPSE due to RA contraction alone) and $\mathrm{TAPSE}_{\mathrm{RV}}$ (RV displacement occurring without the influence of RA contraction) were compared between the two groups [11] (Fig. 1 ).

\subsection{Speckle tracking}

Objects were acquired using a $5-\mathrm{MHz}$ or $7 \mathrm{-MHz}$ probe on a GE Healthcare Japan E9 or E90 system . The analysis was performed using GE Healthcare Japan Echo PAC. The mean values were calculated [1215]; further, the preoperative and postoperative right ventricular free-wall longitudinal strain (RV free-wall longitudinal strain), peak RA longitudinal strain (PRALS), and peak left atrial peak longitudinal strain of the four-chamber views were compared by calculating the average of the strain values for each segment. Right atrial longitudinal strain was classified into right atrial reservoir strain (RA reservoir strain), right atrial conduit strain (RA conduit strain), and right atrial booster strain (RA booster strain) (Fig. 2 ).

\subsection{Statistical analysis}

The values for each group are presented as median (interquartile range). Analyses were performed using SPSS Statistics for Windows (version 27.0; SPSS Japan Inc., Tokyo, Japan). The Mann-Whitney U test and Fisher's exact test were used to compare the two groups. Multivariate logistic regression analysis was performed to identify independent echocardiographic parameters for the presence of post-PH. Receiver operating characteristic (ROC) curve analysis was performed to assess the accuracy of echocardiographic 
measurements for preoperative RV function estimating postoperative PH. Two-tailed p-values of $<0.05$ indicated statistical significance.

3. Results3.1 Preoperative background characteristics between the two groups

The post-PH group included 10 patients and the post-NPH group included 45 patients. There were no significant differences between the two groups in terms of patient background characteristics except for body weight and the proportion of DS patients. The post-PH group had a significantly lower body weight (4.5 $\mathrm{kg}$ ) than the post-NPH group $(5.3 \mathrm{~kg})$. The proportion of patients with DS in the post-PH and post-NPH groups was $70.0 \%$ and $26.7 \%$, respectively, with a significant difference between the two groups. (Table. 1)

\subsection{Comparison of preoperative echocardiographic measurements in two groups}

There were no significant differences between the groups in terms of echocardiographic measurements of the left atrial and ventricular systems. Post-PH patients had a significantly lower TAPSE $(11.1 \mathrm{~mm})$ than post$\mathrm{NPH}$ patients $(14.1 \mathrm{~mm})$. In terms of TAPSE content, post-PH patients had a significantly lower TAPSE $\mathrm{RA}$ $(7.0 \mathrm{~mm})$ than post-NPH patients $(8.7 \mathrm{~mm})$, while there were no significant differences in TAPSE $\mathrm{RV}$. PostPH patients had significantly lower PRALS $(32.0 \%)$ than post-NPH patients $(43.0 \%)$. There were also no significant differences in the VSD dimension and VSD PG between the two groups. Post-PH patients also had significantly lower RA total EF and RA expansion index $(36.1 \%, 56.4 \%$, respectively) than post-NPH patients $(41.5 \%, 70.8 \%$, respectively). (Table 2)

Regarding RA longitudinal strain, post-PH patients had a significantly lower RA reservoir and booster strains $(32.0 \%$ and $19.0 \%$, respectively) than post-NPH patients (43.0\% and $29.0 \%$, respectively). (Table 3) (Fig. 3)

Multivariate logistic regression model demonstrated that PRALS was independent risk factor for the presence of post-PH. (Table 4)

3.3 Accuracy of preoperative parameters in estimating postoperative $\mathrm{PH}$

According to the ROC curve, the PRALS [?] $35 \%$ had the $88.9 \%$ of sensitivity and $70.0 \%$ of specificity of predicting post-PH with a highest area under the curve (AUC) of 0.85 than others ( $\mathrm{p}<0.01,95 \%$ confidence interval [CI]: 0.87 - 0.99). (Fig.4)

\section{Discussion}

Postoperative PH due to VSD is generally associated with delayed cardiac surgery and residual pulmonary vascular occlusive lesions [16]. In particular, among patients with DS, those with left-to-right shunt diseases, such as a VSD or an atrioventricular septal defect, are at an increased risk of postoperative PH [17]. In recent years, surgery has often been performed in early infancy before worsing PH. However, postoperative $\mathrm{PH}$ is often a complication of major VSDs that occur even in these patients. Postoperative PH is associated with RV failure $[18,19]$ and individuals with high pulmonary artery resistance and low pulmonary vascular compliance are associated with postoperative $\mathrm{PH}[20]$. In our study, post-PH patients had mild to moderate $\mathrm{PH}$ with 27-47 mmHg of postoperative TRPG, but not severe. Rheumatoid arthritis patients with mild PH worse RV function measured by TAPSE than control [21]. An adult PH study showed that RV dysfunction occurs when pulmonary artery pressure is mild $(>27 \mathrm{mmHg})$ [22]. These indicated we should be careful to the risk of even postoperative mild to moderate $\mathrm{PH}$ which can cause RV dysfunction. However, it is difficult to preoperatively predict whether a patient with a large VSD will have a high pulmonary artery resistance, and consequently develop postoperative $\mathrm{PH}$. We believe that it would be best to predict postoperative $\mathrm{PH}$ using a non-invasive method. Therefore, in this study, we examined the parameters of preoperative echocardiography for VSD.

RV afterload resulting from pulmonary vascular occlusive lesions usually presenting in patients with DS can cause a decrease in RV function [6,23]. Our study showed that some parameters of preoperative RV function, including RA total EF, RA expansion index, RA longitudinal strain (reservoir and booster strain) 
and TAPSE (especially TAPSE $\mathrm{RA}_{\mathrm{A}}$ ), were decreased in the post-PH group compared to those in the post-NPH group.

The mean value of the maximal atrial strain generally reflects ventricular end-diastolic pressure [24], and a significant inverse correlation with ventricular end-diastolic pressure has been shown in children and those with congenital heart disease $[25,26]$. RA function is a useful index for evaluating RV diastolic function in $\mathrm{PH}[27,28]$ and has been reported to have a significant negative correlation with pulmonary artery systolic pressure and pulmonary vascular resistance, indicating that the RA strain is directly related to the severity of PH [29, 30]. After an ASD is corrected using open-heart surgery, decreased RA strain is also associated with decreased RV diastolic stiffness [31]. Right atrial reservoir function decreases over time from mild RV maladaptation to severe RV maladaptation in $\mathrm{PH}[32]$ and is associated with RAP and mPAP in PH patients $[33,34]$. RA conduit and pump function were also related to the severity of $\mathrm{PH}$ and RV diastolic dysfunction [35]. In our study, we also found that RA reservoir and booster strains decreased in post-PH, but did not RA conduit strain. Another recent study showed that RA reservoir strain is only correlated with mPAP, although RA conduit and booster strains are not [36]. Therefore, the RA reservoir function could change more than other RA strains in PH. Our study showed that PRALS of [?] $35 \%$ is the useful for detecting preoperative $\mathrm{PH}$ because of its high accuracy and AUC, and it may be the most sensitive parameter that reflects the possibility of post-PH preoperatively.

TAPSE has been regarded as an index of RV contractility [37], and its relationship with RV diastolic stiffness and RA function has been clarified [11,38]. In the left ventricular system, mitral annular plane systolic excursion is also related to LV diastolic dysfunction [39]. In particular, TAPSE $\mathrm{RA}_{\mathrm{R}}$, which is TAPSE in the diastole phase, indicates the RA function of contractility [11]. Our study showed that TAPSE RA was decreased in post-PH patients, although TAPSE $_{\mathrm{RV}}$ was not different between the two groups. This indicated that RA contractility was impaired in post-PH patients postoperatively, while RV systolic function was not. RA EF and RA expansion index are useful for evaluating RV diastolic function and the severity of PH [40]. Our study also showed that RA EF and RA expansion index were lower in the post-PH group. We proved that RA function was impaired, and RV diastolic function decreased in VSD patients with post-PH. Right ventricular diastolic stiffness increased in patients with idiopathic PH because of progressive pathologic RV hypertrophy and collagen deposition in the RV tissue [41-44]. Right ventricular diastolic dysfunction precedes RV systolic dysfunction in patients with $\mathrm{PH}$, evaluating RV diastolic dysfunction by echocardiography can predict disease progression [45]. In the early stages of $\mathrm{PH}$, contractility is preserved [46]. Our study showed that RV contractile capacity was preserved in post-PH in a similar condition in the early stages of pulmonary hypertension, possibly.

In our study, the body weight of post-PH patients was lower than that of post-NPH in the univariate analysis and post-PH group had a higher proportion of patients with Down syndrome than post-NPH groups. $30 \%$ of patients with DS involved permanent pulmonary vascular occlusive lesions [22]. However, the lower body weight and DS were confounded with PRALS in multivariate analysis. Patients with the lower body weight mostly might result from conplicating DS. Therefore, preoperative RV dysfunction should be considered in patients with VSD, regardless of the DS status.

Little is known about preoperative RA and RV function in VSD patients. Based on our study, preoperative PRALS could be useful factors for predicting postoperative PH.

\section{Limitations}

The main limitations of this study include its cross-sectional retrospective design and the small number of enrolled patients. It is important to conduct a large prospective comparative study to more accurately evaluate RV function in the future. Second, other parameters of RV systolic and diastolic function, such as pulse-Doppler at the tricuspid valve E and A wave velocity, E', A, and S velocity of tissue Doppler, threedimensional RV ejection fraction, and total ejection isovolume index, were neither measured nor examined in this study. Even though we were able to examine RV systolic and diastolic function at a minimum, a study using more parameters is necessary to verify preoperative RV function in detail. Third, we mainly 
used the TRPG-estimated pulmonary artery pressure on echocardiography for the diagnosis of PH, which may be less accurate than catheterization. However, since TRPG has been proven to be highly correlated with pulmonary artery systolic pressure [10], it is not expected to be a major problem in this study.

5. Conclusion

The RV diastolic function was already impaired prior to surgery in some patients with VSD during early infancy. During surgical management of these patients, attention should be given to the possible development of postoperative PH. We believe that our study may be useful because evaluating PRALS may allow pediatric cardiologists to stratify the risk of VSD patients with pulmonary vascular occlusive lesions.

\section{Authors' contributions:}

Conceptualization: Junpei Kawamura, Methodology: Junpei Kawamura, Kentaro Ueno, Formal analysis and investigation: Junpei Kawamura, Kentaro Ueno, Tsubasa Shimozono, Yoshihiro Takahashi, Koji Nakae, Writing - original draft preparation: Junpei Kawamura,Writing - review and editing: Kentaro Ueno, Yasuhiro Okamoto, Critical revision of article: Tsubasa Shimozono, Yoshihiro Takahashi, Koji Nakae, Kentaro Ueno, Yasuhiro Okamoto, Approval of article, Supervision: Yasuhiro Okamoto .

\section{References}

[1] R. Formigari, R.M. Di Donato, G. Gargiulo, et al, Better surgical prognosis for patients with complete atrioventricular septal defect and Down's syndrome. Ann. Thorac. Surg. 78 (2004) 666-72; discussion 672. DOI: $10.1016 / \mathrm{j}$.athoracsur.2003.12.021.

[2] Michele D'Alto, Vaikom S Mahadevan. Pulmonary arterial hypertension associated with congenital heart disease. Eur Respir Rev. 2012;21(126):328-37. DOI: 10.1183/09059180.00004712.

[3] M. Sungur, B. Ocal, D. Oğuz, et al. Plasma endothelin-1 and nitrate levels in Down's syndrome with complete atrioventricular septal defect-associated pulmonary hypertension: A comparison with non-Down's syndrome children. Eur. J. Pediatr. 168 (2009) 593-597. DOI: 10.1007/s00431-008-0803-1.

[4] N. Galiè, M. Humbert, J.L. Vachiery, et al, ESC Scientific Document Group , 2015 ESC/ERS Guidelines for the diagnosis and treatment of pulmonary hypertension: The Joint Task Force for the Diagnosis and Treatment of Pulmonary Hypertension of the European Society of Cardiology (ESC) and the European Respiratory Society (ERS): Endorsed by: Association for European Paediatric and Congenital Cardiology (AEPC), International Society for Heart and Lung Transplantation (ISHLT). Eur. Heart J. 37 (2016) 67-119. DOI: $10.1093 /$ eurheartj/ehv317.

[5] S. Rosenkranz, H.A. Ghofrani, M. Beghetti, et al, Riociguat for pulmonary arterial hypertension associated with congenital heart disease. Heart. 101 (2015) 1792-1799. DOI: 10.1136/heartjnl-2015-307832.

[6] P.R. Forfia, J.L. Vachiéry, Echocardiography in pulmonary arterial hypertension. Am. J. Cardiol. 110 (Suppl.) (2012) 16S-24S. DOI: 10.1016/j.amjcard.2012.06.012.

[7] H. Amano, S. Abe, S. Hirose, et al, Comparison of echocardiographic parameters to assess right ventricular function in pulmonary hypertension. Heart Vessels. 32 (2017) 1214-1219. DOI: 10.1007/s00380-017-0991-6.

[8] A.M. Smith, P.T. Levy, O. Franklin, et al. Pulmonary hypertension and myocardial function in infants and children with Down syndrome. Arch. Dis. Child. 105(11) (2020) 1031-1034. DOI: 10.1136/archdischild2019-318178.

[9] Colm R Breatnach, Neidin Bussmann, Aisling Smith, et.al. Cardiac mechanics in infants with Down syndrome in the early neonatal period. J Perinatol 39 (2019) 626-633. DOI: 10.1038/s41372-019-0354-5.

[10] Naoko Sawada, Takayuki Kawata, Masao Daimon, et al. Detection of Pulmonary Hypertension with Systolic Pressure Estimated by Doppler Echocardiography. Int Heart J. 60(4) (2019) 836-844. DOI: 10.1536/ihj.18-453. 
[11] Joseph A Sivak, Amresh Raina, Paul R Forfia. Assessment of the physiologic contribution of right atrial function to total right heart function in patients with and without pulmonary arterial hypertension. Pulm Circ. 6 (3) (2016) 322-8. DOI: 10.1086/687767.

[12] M. Padeletti, M. Cameli, M. Lisi, et al, Right atrial speckle tracking analysis as a novel noninvasive method for pulmonary hemodynamics assessment in patients with chronic systolic heart failure. Echocardiography 28 (2011) 658-664. DOI: 10.1111/j.1540-8175.2011.01413.x.

[13] J.U. Voigt, G. Pedrizzetti, P. Lysyansky, et al, Definitions for a common standard for 2D speckle tracking echocardiography: Consensus document of the EACVI/Ase/Industry Task Force to standardize deformation imaging. J. Am. Soc. Echocardiogr. 28 (2015) 183-193. DOI: 10.1016/j.echo.2014.11.003.

[14] Hatice S Kemal, Meral Kayikcioglu, Hakan Kultursay, et al. Right ventricular free-wall longitudinal speckle tracking strain in patients with pulmonary arterial hypertension under specific treatment. Echocardiography. 34 (2017) 530-536. DOI: 10.1111/echo.13472.

[15] Jun Huang, Chao Yang, Cai-Fang Ni, et al. Right atrial function assessed by volume-derived values and speckle tracking echocardiography in patients with hypertrophic cardiomyopathy. BMC Cardiovasc Disord. 20(1) (2020) 335. DOI: 10.1186/s12872-020-01610-1.

[16] S. Ikawa, Y. Shimazaki, S. Nakano, et al. Pulmonary vascular resistance during exercise late after repair of large ventricular septal defects. Relation to age at the time of repair. J. Thorac. Cardiovasc. Surg. 109 (1995) 1218-1224. DOI: 10.1016/S0022-5223(95)70206-7.

[17] T. Saji, Clinical characteristics of pulmonary arterial hypertension associated with Down syndrome. Pediatr. Int. 56 (3) (2014) 297-303. DOI: 10.1111/ped.12349.

[18] B.A. Nasser, A.R. Mesned, T. Mohamad, et al. Incidence and causes of prolonged mechanical ventilation in children with Down syndrome undergoing cardiac surgery. J. Saudi Heart Assoc. 30 (3) (2018) 247-253. DOI: $10.1016 /$ j.jsha.2018.01.004.

[19] N.S. Hill, K.R. Roberts, I.R. Preston. Postoperative pulmonary hypertension: Etiology and treatment of a dangerous complication. Respir. Care 54 (7) (2009) 958-968. DOI: 10.4187/002013209793800439.

[20] Y. Iwaya, J. Muneuchi, Y. Inoue, et al. Relationship between pulmonary arterial resistance and compliance in patients with Down syndrome. Pediatr. Cardiol. 40 (4) (2019) 841-847. DOI: 10.1007/s00246-01902080-9.

[21] Azpiri-Lopez JR, Galarza-Delgado DA, Colunga-Pedraza IJ, et al. Echocardiographic evaluation of pulmonary hypertension, right ventricular function, and right ventricular-pulmonary arterial coupling in patients with rheumatoid arthritis. Clin Rheumatol. 40(7) (2021) 2651-2656. DOI: 10.1007/s10067-020-05544$\mathrm{z}$.

[22] Jessica H Huston, Bradley A Maron, John French, et al. Association of Mild Echocardiographic Pulmonary Hypertension With Mortality and Right Ventricular Function. JAMA Cardiol. 4 (11) (2019) 1112-1121. DOI: $10.1001 /$ jamacardio.2019.3345.

[23] 'D. Bush, C. Galambos, D.D. Ivy, et al. Clinical Characteristics and Risk Factors for Developing Pulmonary Hypertension in Children with Down Syndrome. J. Pediatr. 202 (2018) 212-219.e2. DOI: 10.1016/j.jpeds.2018.06.031'

[24] M. Cameli, S. Sparla, M. Losito, et al. Correlation of left atrial strain and doppler measurements with invasive measurement of left ventricular end-diastolic pressure in patients stratified for different values of ejection fraction, Echocardiography. 33 (2016) 398-405. DOI: 10.1111/echo.13094.

[25] J. Sabatino, G. Di Salvo, C. Prota, et al. Left atrial strain to identify diastolic dysfunction in children with cardiomyopathies, J. Clin. Med. 8 (2019) 1243. DOI: 10.3390/jcm8081243. 
[26] Antonio Vitarelli, Enrico Mangieri, Carlo Gaudio, et al. Right atrial function by speckle tracking echocardiography in atrial septal defect: Prediction of atrial fibrillation. Clin Cardiol. 41(10) (2018) 1341-1347. DOI: $10.1002 /$ clc. 23051 .

[27] 'G. Querejeta Roca, P. Campbell, B. Claggett, et al. Right Atrial Function in Pulmonary Arterial Hypertension, Circ. Cardiovasc. Imaging. 8 (2015) e003521; discussion e003521. DOI: 10.1161/CIRCIMAGING.115.003521'

[28] H.A. Deschle, A. Amenabar, N.A. Casso, et al, Behavior of right atrial strain in high systolic pulmonary artery pressure, Echocardiography. 35 (2018) 1557-1563. DOI: 10.1111/echo.14102.

[29] Khodr Tello , Antonia Dalmer, Rebecca Vanderpool, et al. Right ventricular function correlates of right atrial strain in pulmonary hypertension: a combined cardiac magnetic resonance and conductance catheter study. Am J Physiol Heart Circ Physiol. 318 (2020) H156-H164. DOI: 10.1152/ajpheart.00485.2019.

[30] Lena Theres, Anne Hübscher, Karl Stangl, et al. Associations of 2D speckle tracking echocardiographybased right heart deformation parameters and invasively assessed hemodynamic measurements in patients with pulmonary hypertension. Cardiovasc Ultrasound. 18 (2020) :13. DOI: 10.1186/s12947-020-00197-z.

[31] O. Ozturk, U. Ozturk, Sengul Ozturk. Assessment of right atrial function with speckle tracking echocardiography after percutaneous closure of an atrial septal defect. Rev. Port. Cardiol. 36 (12) (2017) 895-900. DOI: 10.1016/j.repc.2017.06.013.

[32] Snigdha Jain, Daniel Kuriakose, Ilaina Edelstein, et al. Right Atrial Phasic Function in Heart Failure With Preserved and Reduced Ejection Fraction. JACC Cardiovasc Imaging. 12 (2019) :1460-1470. DOI: 10.1016/j.jcmg.2018.08.020.

[33] Querejeta Roca G, Campbell P, Claggett B, et al. Right atrial function in pulmonary arterial hypertension. Circ Cardiovasc Imaging 8 (2015) : e003521. DOI:10.1161/CIRCIMAGING.115.003521.

[34] K. Sakata, Y. Uesugi, A. Isaka, et al, Evaluation of right atrial function using right atrial speckle tracking analysis in patients with pulmonary artery hypertension, J. Echocardiogr. 14 (2016) 30-38. DOI: 10.1007/s12574-015-0270-4.

[35] Pei-Ni Jone, Michal Schäfer, Ling Li, et al. Right Atrial Deformation in Predicting Outcomes in Pediatric Pulmonary Hypertension. Circ Cardiovasc Imaging. 10 (2017):e006250. DOI: 10.1161/CIRCIMAGING.117.006250.

[36] Lena Theres, Anne Hübscher, Karl Stangl, et al. Associations of 2D speckle tracking echocardiographybased right heart deformation parameters and invasively assessed hemodynamic measurements in patients with pulmonary hypertension. Cardiovasc Ultrasound. 18(1) (2020) 13. DOI: 10.1186/s12947-020-00197-z.

[37] M Guazzi, F Bandera, G Pelissero, et al. Tricuspid annular plane systolic excursion and pulmonary arterial systolic pressure relationship in heart failure: an index of right ventricular contractile function and prognosis. Am J Physiol Heart Circ Physiol. 305(9) (2013) H1373-81. DOI: 10.1152/ajpheart.00157.2013.

[38] Wojciech Mądry, Maciej Aleksander Karolczak, Marcin Myszkowski. Critical appraisal of MAPSE and TAPSE usefulness in the postoperative assessment of ventricular contractile function after congenital heart defect surgery in infants. J Ultrason. 19(76) (2019) 9-16. DOI: 10.15557/JoU.2019.0002.

[39] Dagmar F Hernandez-Suarez, Francisco Lopez-Menendez, Abiel Roche-Lima, et al. Assessment of Mitral Annular Plane Systolic Excursion in Patients With Left Ventricular Diastolic Dysfunction. Cardiol Res. 10 (2) (2019) 83-88. DOI: $10.14740 /$ cr837.

[40] Silvia Rain, M Louis Handoko, Pia Trip, Cet al. Right ventricular diastolic impairment in patients with pulmonary arterial hypertension. Circulation. 128 (2013) :2016-25, 1-10. DOI: 10.1161/CIRCULATIONAHA.113.001873. 
[41] Ren X, Johns RA, Gao WD. EXPRESS: Right heart in pulmonary hypertension: from adaptation to failure. Pulm Circ. 9 (2019):2045894019845611.

[42] Sagie A, Benjamin EJ, Galderisi M, et al. Echocardiographic assessment of left ventricular structure and diastolic filling in elderly subjects with borderline isolated systolic hypertension (the Framingham Heart Study). Am J Cardiol. 72 (1993) :662-665.

[43] Frangogiannis NG. The extracellular matrix in ischemic and nonischemic heart failure. Circ Res 125 (2019) :117-146.

[44] Youn-Hoa Jung, Xianfeng Ren, Giancarlo Suffredini, et al. Right ventricular diastolic dysfunction and failure: a review. Heart Fail Rev. 19 (2021). DOI: 10.1007/s10741-021-10123-8.

[45] Hunter C Champion, Evangelos D Michelakis, Paul M Hassoun. Comprehensive invasive and noninvasive approach to the right ventricle-pulmonary circulation unit: state of the art and clinical and research implications. Circulation. 120 (2009):992-1007. DOI: .1161/CIRCULATIONAHA.106.674028.

[46] Javier Sanz, Damián Sánchez-Quintana, Eduardo Bossone, et al. Anatomy, Function, and Dysfunction of the Right Ventricle: JACC State-of-the-Art Review. J Am Coll Cardiol. 73 (2019):1463-1482. DOI: 10.1016/j.jacc.2018.12.076.

\section{Figure Legends}

Figure 1. M-mode tracking of the tricuspid annulus in a patient. TAPSE $\mathrm{RA}_{\mathrm{A}}$ accounts for the proportion of TAPSE owed to RA function alone; TAPSE $E_{R V}$ accounts for RV shortening without the influence of RA contraction.

TAPSE = Tricuspid Annular Plane Systolic Excursion; $R A=$ Right Atrium; $R V=$ Right Ventricle.

Figure 2. Speckle tracking analysis of atrial and ventricular strain.

An apical 4-chamber view of the RV (upper left), LA (upper right) and RA (lower) longitudinal strains were obtained for each patient. Dotted white curve indicates mean of all segments. The maximum excursion of the average strain curve symbolizes the Peak LA longitudinal strain (PLALS). The maximum excursion of the average strain curve symbolizes the Peak RA longitudinal strain and reservoir function (PRALS, RA reservoir strain). RA conduit function was calculated from the difference between the peak RA strain and the end of the conduit phase in early diastole (RA conduit strain) and RA booster function was derived from the difference between the endpoint of conduit phase and the peak RA contraction (RA booster strain).

$R V=$ Right Ventricle; LA=Left Atrium; RA=Right Atrium.

Figure 3. Comparison each values of RA longitudinal strain between post-PH patients and post-NPH patients at reservoir, conduit and contraction phase.

$R A=$ Right Atrium; $P H=$ Pulmonary Hypertension NPH $=$ No Pulmonary Hypertension.

Figure 4. Receiver operating characteristic curve of the PRALS, RA booster strain, RA EF, RA expansion

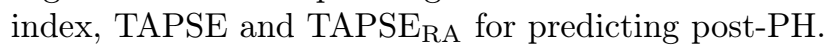

$R A=$ Right Atrium; PRALS $=$ Peak RA longitudinal strain EF $=$ Ejection Fraction; TAPSE $=$ Tricuspid Annular Plane Systolic Excursion; PH = Pulmonary Hypertension.

\section{ACKNOWLEDGMENTS: None.}

FINANCIAL DISCLOSURE: The authors received no financial support for the research, authorship, and/or publication of this article.

CONFLICT OF INTEREST: None declared. 


\section{Hosted file}

Table 1 8.14.docx available at https://authorea.com/users/435983/articles/538637-predictingpostoperative-hypertension-among-patients-with-ventricular-septal-defect-in-infants

\section{Hosted file}

Table 2 8.14.docx available at https://authorea.com/users/435983/articles/538637-predictingpostoperative-hypertension-among-patients-with-ventricular-septal-defect-in-infants

\section{Hosted file}

Table 3 8.14.docx available at https://authorea.com/users/435983/articles/538637-predictingpostoperative-hypertension-among-patients-with-ventricular-septal-defect-in-infants

\section{Hosted file}

Table 4 8.14.docx available at https://authorea.com/users/435983/articles/538637-predictingpostoperative-hypertension-among-patients-with-ventricular-septal-defect-in-infants
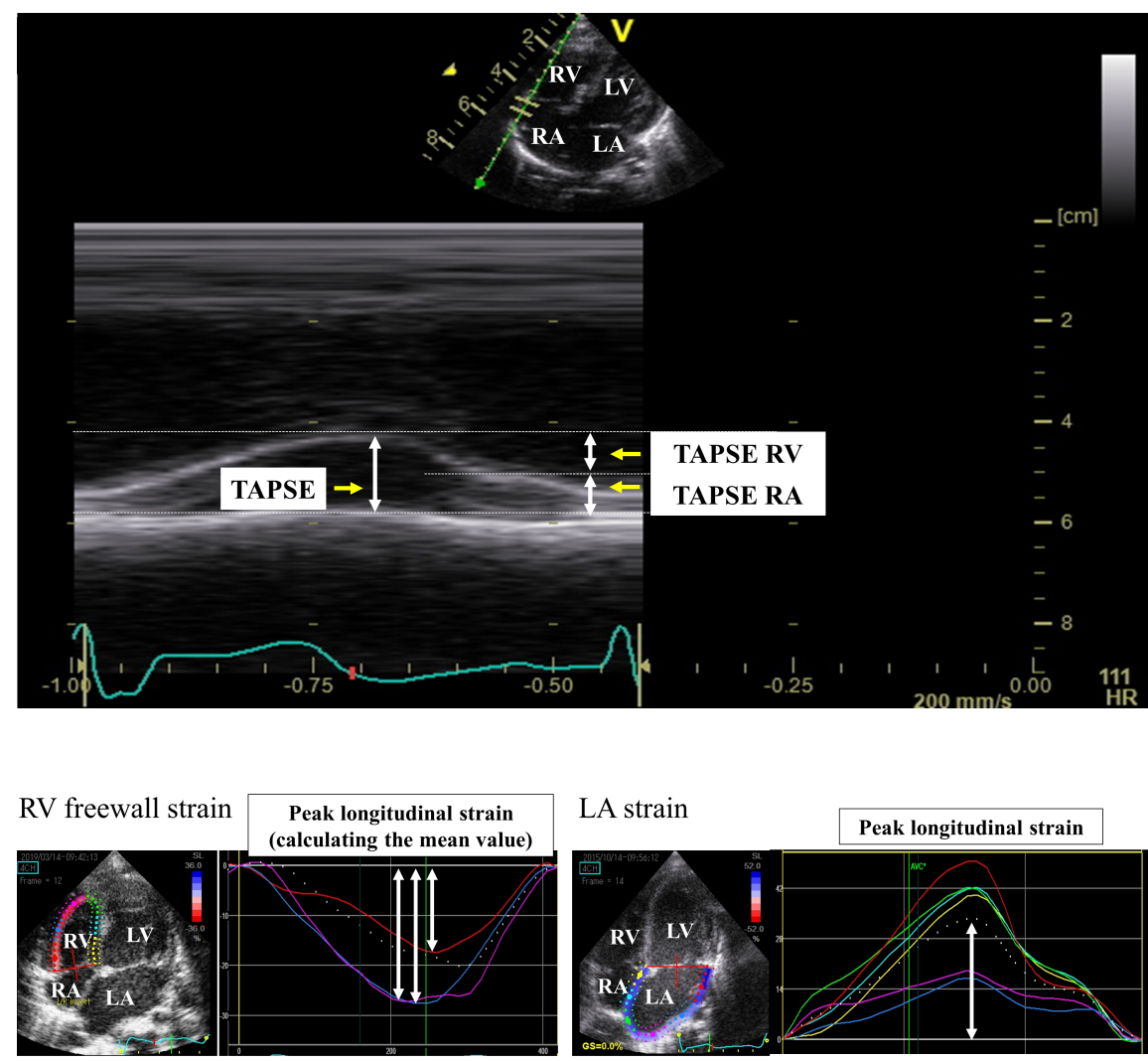

RA strain

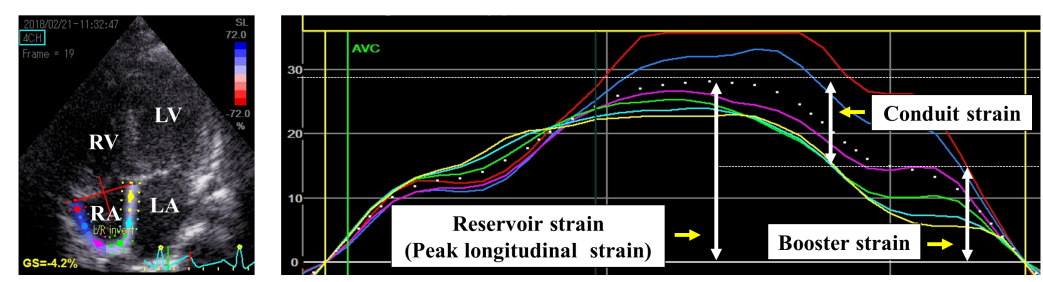



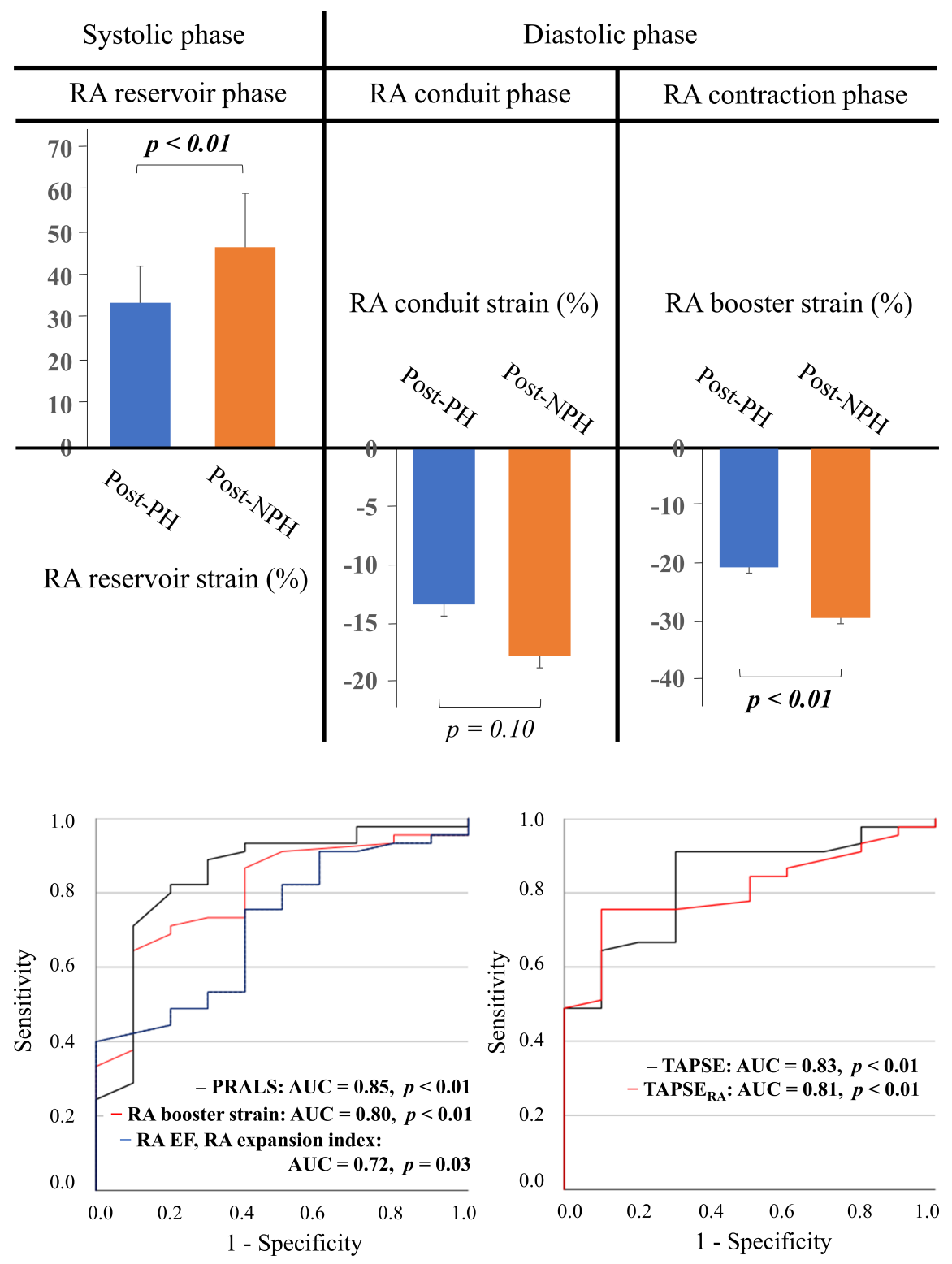